\title{
Association between functional TERT promoter polymorphism rs2853669 and cervical cancer risk in South Indian women
}

\author{
VILVANATHAN VINOTHKUMAR ${ }^{1}$, KANAGARAJ ARUN $^{1}$, GANESAN ARUNKUMAR $^{1}$, \\ SUNDARAMOORTHY REVATHIDEVI ${ }^{1}$, RAJENDREN RAMANI ${ }^{2}$, LAKKAKULA V.K.S. BHASKAR ${ }^{3}$, \\ AVANIYAPURAM KANNAN MURUGAN ${ }^{4}$ and ARASAMBATTU KANNAN MUNIRAJAN ${ }^{1}$ \\ ${ }^{1}$ Department of Genetics, Dr ALM Post Graduate Institute of Basic Medical Sciences, University of Madras, \\ Chennai, Tamil Nadu 600113; ${ }^{2}$ Institute of Social Obstetrics and Government Kasturba Gandhi \\ Hospital for Women and Children, Chennai, Tamil Nadu 600005; ${ }^{3}$ Sickle Cell Institute Chhattisgarh \\ (SCIC), Raipur, Chhattisgarh 492001, India; ${ }^{4}$ Department of Molecular Oncology, King Faisal \\ Specialist Hospital and Research Centre, Riyadh 11211, Kingdom of Saudi Arabia
}

Received December 20, 2018; Accepted July 17, 2019

DOI: $10.3892 / \mathrm{mco} .2020 .2003$

\begin{abstract}
A single nucleotide polymorphism (SNP) rs2853669 $(A>G)$ in the telomerase reverse transcriptase (TERT) promoter has recently been reported in chr5:1,295,349 T>C (T349C), and was shown to be associated with increased cancer risk and poor survival in a specific population. However, at present, the role of this particular SNP with TERT promoter driver mutations and its genetic association with human papilloma virus (HPV) in patients with cervical cancer has not been determined. In the present study, the genetic association of the functional SNP rs2853669 in the presence/absence of TERT promoter hotspot mutations and HPV in patients with cervical cancer of South Indian origin was evaluated. To understand and compare the frequency of the variant allele and its risk association in different cancer types of various populations, the SNP was genotyped in 257 cervical cancer samples and 295 controls, and its associations with TERT promoter hotspot mutations and HPV were analyzed. Furthermore, an extensive search of previously published articles in PubMed, Embase and Web of Science was conducted; a meta-analysis was carried out to elucidate the association of the SNP with different cancer types in global populations. The SNP analysis
\end{abstract}

Correspondence to: Professor Arasambattu Kannan Munirajan, Department of Genetics, Dr ALM Post Graduate Institute of Basic Medical Sciences, University of Madras, Taramani Campus, Taramani, Chennai, Tamil Nadu 600113, India

E-mail: akmunirajan@gmail.com

Abbreviations: HPV, human papilloma virus; TERT, telomerase reverse transcriptase; ETS, E-twenty six

Key words: human papilloma virus, telomerase reverse transcriptase, cervical cancer, promoter single nucleotide polymorphism, rs2853669, polymorphism showed significantly high frequency (41\%) of homozygous variant allele rs2853669 (GG) in patients with cervical cancer compared with control samples [Recessive allele model odds ratio $(\mathrm{OR})=1.71 ; 95 \% \mathrm{CI}=1.20-2.43 ; \mathrm{P}=0.003]$. No significant interaction was observed between the TERT SNP rs2853669 and HPV status as well as other hotspot TERT promoter (C228T and C250T) mutations determined in our previous study. In addition, the overall meta-analysis revealed a significant association of the SNP rs2853669 with other cancer types in different ethnic populations $(\mathrm{OR}=1.09$; 95\% $\mathrm{CI}=1.03-1.16$; $\mathrm{P}=0.004)$. The present results suggested that the TERT SNP rs2853669 could play an important role in the risk of cervical cancer in a South Indian population.

\section{Introduction}

Telomeres are special structures at the ends of the chromosomes consisting of 'TTAGGG' tandem repeat sequences along with its associated protein complex called 'shelterin' (1). As a result of the inability of DNA polymerases to duplicate the ends of the linear DNA molecules, the lengths of the human telomeres get shortened to $\sim 50 \mathrm{bp}$ with each cell division and the attrition of telomere length is counteracted by telomerase reverse transcriptase (TERT), which is the catalytic component of the enzyme telomerase (2). It has been reported that TERT is reactivated in cancer cells and was found to be overexpressed in tumors (3). Several mechanisms are associated with TERT reactivation, including TERT promoter mutations or rearrangements, and copy number amplification and methylation (4). Cancer cells survive by exploiting the telomere maintenance mechanism. The details of cellular immortality through the telomere length maintenance are poorly understood in many human cancer types, including cervical cancer.

The prevalence of cervical cancer has increased rapidly in rural areas and overall it ranks second in both incidence and mortality rates in India, regardless of its incidental difference between rural and urban areas (5). In total, 95\% of cases are caused by persistent infection with high-risk human papilloma 
virus (HPV) (6). Persistent HPV infection and viral oncogene expression results in the inactivation of tumor suppressor genes, including tumor protein 53 and $\mathrm{pRb}$, that consequently leads to increased genomic instability and accumulation of mutations, which often results in tumorigenesis (7). Cervical cancer remains a serious problem among women, especially in developing nations like India even after several decades of cervical cancer research that has identified various therapeutic regiments.

Recent advancement in next generation sequencing has enabled the whole genome sequencing of tumors with their paired normal controls. The Cancer Genome Atlas and International Cancer Genome Consortium studies revealed significant non-coding mutations and single nucleotide polymorphism (SNPs) in the regulatory regions of genes associated with tumorigenesis. The pan-cancer analysis of the whole genome aimed to analyze whole genomes of 2,500 tumors and matched normal controls, mainly to identify driver mutations and to differentiate from passenger mutations (8). The previously identified driver mutations (C228T and C250T) in the TERT promoter were found to create a new binding site for the E-twenty six (ETS) family of transcription factors, resulting in the increased expression of TERT, which was initially observed in melanomas $(9,10)$ and later in other cancer types (11). A previous study also observed the same functional TERT promoter mutations with high frequency $(21.4 \%)$ in South Indian cervical cancer (12).

Previously, an SNP rs2853669 (A>G) in the TERT promoter was identified in chr5:1,295,349 T>C (T349C) and was shown to be associated with cancer risk in a different population (13-15). The presence of the SNP rs2853669 along with reactivating promoter mutations has been reported to increase the risk and shown to be associated with a poor survival rate in hepatocellular carcinoma (16). At present, the role of this particular SNP with TERT promoter driver mutations and its genetic association with HPV in patients with cervical cancer has not been determined. To understand the genetic association of the TERT SNP with cervical cancer risk, the present study focused on analyzing the SNP rs2853669 in cervical cancer samples from South Indian women and healthy controls. Furthermore, a meta-analysis of the SNP rs2853669 in various cancer types of world populations was conducted to elucidate the distribution and risk association of variant alleles with different types of cancer.

\section{Materials and methods}

Study design and subjects. The present study was conducted after obtaining approval from the Institutional Ethics Committee (approval no. 04092010) of Madras Medical College and Hospital (Chennai, India). The present study was conducted between January 2011 and June 2015. The tissue/blood samples were collected at The Institute of Social Obstetrics and Government Kasturba Gandhi Hospital for Women and Children (Chennai, India) and Government Royapettah Hospital (Chennai, India) after obtaining written informed consent from each patient. The present study included tissue samples from 257 patients with cervical cancer confirmed by histologic examination of biopsies and curettage specimens in pathology laboratory. For histologic classification, a two-tiered cervical intraepithelial neoplasia system was employed (17). The samples with no reported malignancy in the pathology were excluded. The biopsy samples were collected during the diagnosis of the patients and transported to the laboratory in RNAlater solution (Thermo Fisher Scientific, Inc.). The transported samples were homogenized and stored in RNAlater solution at $4^{\circ} \mathrm{C}$ overnight. On the subsequent day, RNAlater solution was discarded and DNA was isolated from the tissues after PBS washes following a standard phenol-chloroform extraction method. In total, 295 healthy women were recruited as controls. About $2 \mathrm{ml}$ of blood was collected in EDTA-coated vacutainers by venipuncture of the dorsal hand veins. Blood samples were also collected from patients with cervical cancer to sequence them to confirm the germline nature of the TERT SNP. The mean age of the patients and controls were 51.2 \pm 11.3 and $39.41 \pm 10.6$ years, respectively. DNA was isolated from all the blood samples using a standard phenol-chloroform extraction and ethanol precipitation method (18).

SNP genotyping. To analyze the frequency of the SNP rs2853669 $A>G$ in patients with cervical cancer and the controls, a hydrolysis probe-based allelic discrimination assay was performed (Thermo Fisher Scientific, Inc.). The PCR reaction was carried out in a total of $5 \mu \mathrm{l}$ comprised of $10 \mathrm{ng}$ DNA and $2.5 \mu 12 \mathrm{X}$ TaqMan Universal PCR master mix (No UNG) and $0.125 \mu \mathrm{l} 40 \mathrm{X}$ TaqMan SNP genotyping assay mix (Assay ID: C-8773290_10; Thermo Fisher Scientific, Inc.). The reaction was performed in QuantStudio 6 Flex Real-Time PCR (Thermo Fisher Scientific, Inc.) using a standard protocol $\left(2 \mathrm{~min}\right.$ at $50^{\circ} \mathrm{C}, 10 \mathrm{~min}$ at $95^{\circ} \mathrm{C}$ followed by $15 \mathrm{sec}$ at $92^{\circ} \mathrm{C}$ and $60 \mathrm{sec}$ at $60^{\circ} \mathrm{C}$ for 40 cycles) and the allelic discriminations were conducted by detecting the fluorescence in the PCR reactions. A control with no template was included in each plate. Genotype calls of $95 \%$ quality were scored using Sequence Detection Software v2.4.1 (Thermo Fisher Scientific, Inc.).

DNA sequencing. The germline nature of the identified TERT SNP was confirmed by sequencing $2 \%$ parallel blood DNA available from the patients with cervical cancer and the controls. The human TERT promoter region was amplified from cervical cancer tissue DNA samples and sequencing was performed, as previously described (12). The promoter-specific primers TERT forward: 5'-TGTAAAACGACGGCCAGT GGCCGATTCGACCTCTCTC-3' and reverse: 5'-CAGCGC TGCCTGAAACTCG-3' (the underlined sequence in the TERT $F$ is the M13 universal sequencing primer) were used to amplify the TERT promoter. Briefly, the thermocycling conditions for PCR were $5 \mathrm{~min}$ at $95^{\circ} \mathrm{C}$ once followed by $30 \mathrm{sec}$ at $95^{\circ} \mathrm{C}, 45 \mathrm{sec}$ at $60^{\circ} \mathrm{C}$, and $45 \mathrm{sec}$ at $72^{\circ} \mathrm{C}$ for 10 cycles and 30 cycles of $30 \mathrm{sec}$ at $95^{\circ} \mathrm{C}, 45 \mathrm{sec}$ at $60^{\circ} \mathrm{C}$ and $30 \mathrm{sec}$ (with $5 \mathrm{sec}$ increases in each cycle) at $72^{\circ} \mathrm{C}$ and final extension for $7 \mathrm{~min}$ at $72^{\circ} \mathrm{C}$. The PCR products were purified using a commercial kit (Qiagen, Inc.) and sequenced by the Sanger sequencing method (Macrogen, Inc.). The representative sequence for all three genotypes is presented in Fig. S1.

Statistical analysis. The genotype and allele frequency of the cervical cancer and control groups were estimated using the gene count method. Hardy-Weinberg Equilibrium (HWE) 


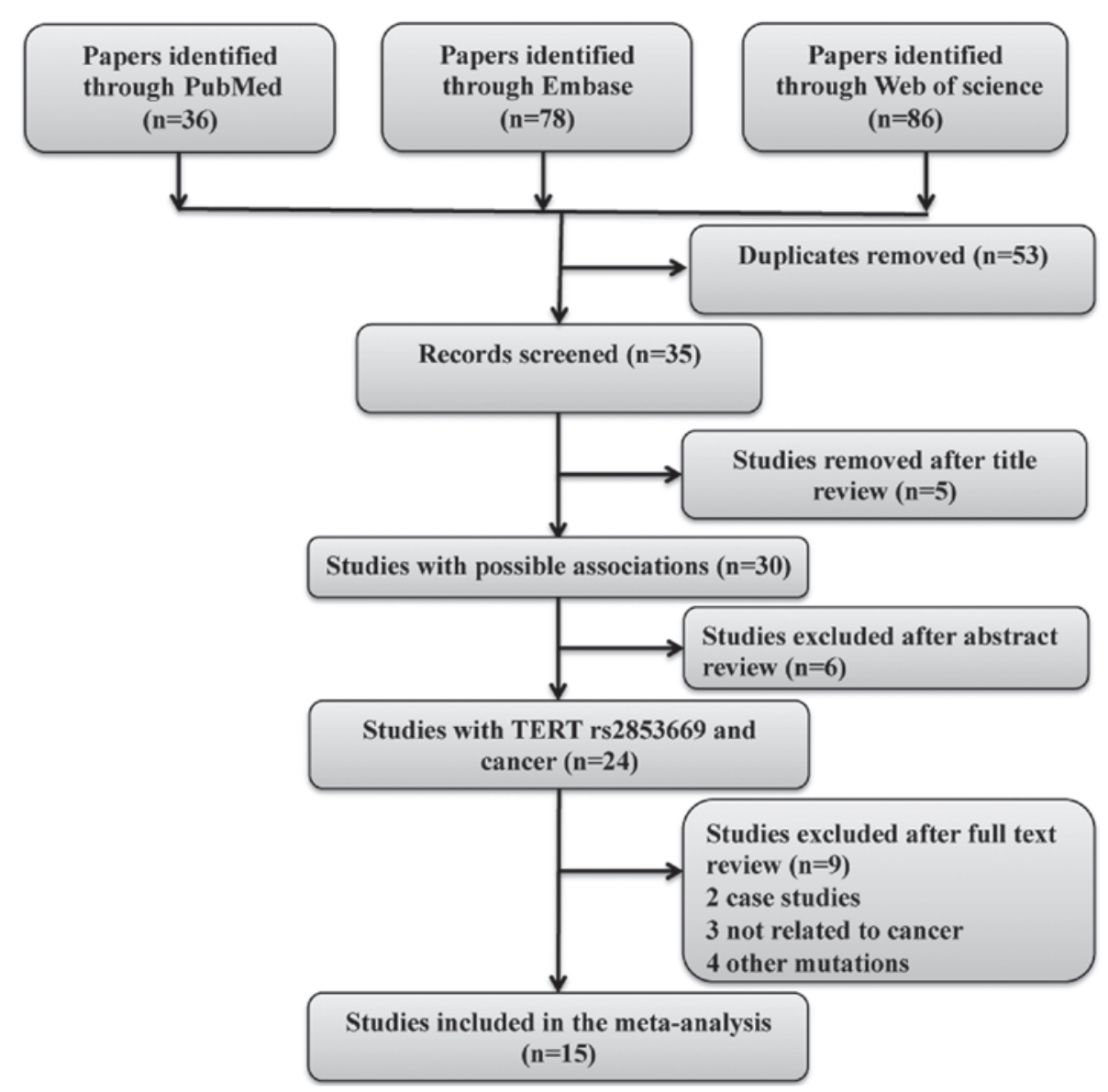

Figure 1. Flow chart shows the selection of eligible studies included in the meta-analysis. TERT, telomerase reverse transcriptase.

was assessed by the goodness-of-fit $\chi^{2}$ test (19). The association between the polymorphism and cancer was analyzed using a $\chi^{2}$ test. The odds ratios (ORs) with $95 \%$ CIs were calculated to evaluate the association between the rs2853669 polymorphism and cervical cancer risk, including variant heterozygous vs. wild-type homozygous model, and variant homozygous vs. wild-type homozygous model and dominant model. All tests were two-tailed and $\mathrm{P}<0.05$ was considered to indicate a statistically significant difference. All the analyses were performed using SPSS, version 20.0. (IBM, Corp.).

Meta-analysis. Meta-analysis was performed to identify the distribution of rs2583669 variant allele across various types of cancers among different populations. An extensive literature search wasconducted using PubMed(https://www.ncbi.nlm.nih. gov/pubmed/), Embase (https://www.embase.com/) and Web of Science (https://clarivate.com/products/web-of-science/) databases (last search date January 10, 2019). The key words used in the search included 'TERT or telomerase reverse transcriptase', 'cancer or carcinoma' and 'rs2583669 polymorphism'. After the initial screening, the full text of all relevant papers was obtained and further filtered in order to fit into the inclusion criteria: i) Studies examining the association of TERT rs2583669 polymorphisms with cancer; ii) prospective case-control studies; and iii) studies with information to calculate ORs. Then, two authors independently extracted the genotype frequencies of the TERT rs2583669 polymorphism. This meta-analysis evaluated the association of TERT rs2583669 polymorphism with cancer risk according to the guidelines issued in the Preferred Reporting Items for Systematic Reviews and Meta-Analyses statement (20). A flow-chart describing the present study and the selection criteria for the meta-analysis is presented in Fig. 1. All meta-analyses were conducted using OpenMetaAnalyst software (21). All the previous studies included in the meta-analysis were evaluated for the HWE using a $\chi^{2}$ test in the control groups and $\mathrm{P}>0.05$ was considered as the sample neutrality. The carrier risk associations for rs2853669 were calculated in the allelic, dominant and recessive models using $\mathrm{R}$ (version 3.2.0) statistical software (https://cran.r-project. org/bin/windows/base/old/3.2.0/). The OR and 95\% CIs were illustrated using Forest plot graphical representation. Furthermore, a sensitivity analysis was conducted by excluding one study in each analysis to examine the robustness of the method used for the meta-analysis. The potential publication bias was assessed by Begg's funnel plot and Egger's test (22).

\section{Results}

Frequency of rs2853669 in South Indian cervical cancer. In the present study, the promoter SNP rs2853669 $(-245 \mathrm{~A}>\mathrm{G})$ was genotyped in 552 samples, consisting of 257 cervical cancer samples and 295 controls from South India. The distribution of alleles along with various clinical variables is presented in Table I. The proportions of genotypes were $23.3 \%$ AA, $35.4 \% \mathrm{AG}$ and $41.3 \% \mathrm{GG}$ in the cervical cancer cases, and 
Table I. Clinicopathological characteristics among the rs2853669 genotypes in patients with cervical cancer.

\begin{tabular}{lccc}
\hline & & & SNP rs2853669 \\
\cline { 3 - 4 } Clinicopathological characteristics & Total $(\%)$ & AA $(\%)$ & AG+GG $(\%)$ \\
\hline No. of cases & 257 & $60(23.3)$ & $197(76.7)$ \\
Age in years (mean \pm SD) & $51.2 \pm 11.3$ & & \\
$<51$ & $138(53.7)$ & $29(21.1)$ & $109(78.9)$ \\
$\geq 51$ & $116(45.1)$ & $31(26.7)$ & $85(73.3)$ \\
Unknown & $3(1.2)$ & - & $3(100)$ \\
Tumor cell differentiation & & $11(20.4)$ & $43(79.6)$ \\
Well differentiated & $54(21)$ & $34(29.4)$ & $82(70.6)$ \\
Moderately differentiated & $116(45.1)$ & $10(19.3)$ & $42(80.7)$ \\
Poorly differentiated & $52(20.2)$ & $5(14.7)$ & $30(85.7)$ \\
Unknown & $35(13.6)$ & $6(35.3)$ & $11(64.7)$ \\
CIN grade & & $18(20.9)$ & $68(79.1)$ \\
IB1 or IB2 & $17(6.6)$ & $17(29.3)$ & $41(70.7)$ \\
II or IIA2 and IIB & $86(33.5)$ & - & $3(100)$ \\
III or IIIB & $58(22.6)$ & $19(20.4)$ & $74(79.6)$ \\
IV or IVB & $3(1.2)$ & $50(24.1)$ & $158(75.9)$ \\
$\quad$ Unknown & $93(36.2)$ & & \\
Infiltration & & & \\
Infiltrinfiltrated & $208(80.9)$ & &
\end{tabular}

SNP, single nucleotide polymorphism; AA, wild-type; GG+AG, carrier allele; CIN, cervical intraepithelial neoplasia.

$21.4 \%$ AA, $49.5 \%$ AG and $29.1 \%$ GG in the controls (Table II). A higher incidence [41.25\% (106/257)] of homozygous variant 'GG' was identified in the cervical cancer samples compared with the controls $[29.15 \%(86 / 295)]$ in the study population, although without any statistical significance $(\mathrm{P}=0.265)$. The TERT rs2853669 polymorphism showed significant association with cervical cancer in the recessive model (GG vs. $\mathrm{AA}+\mathrm{AG} ; \mathrm{OR}=1.71 ; 95 \% \mathrm{CI}=1.20-2.43 ; \mathrm{P}=0.003$; Table II). The distribution of TERT rs2853669 polymorphism among TERT activating promoter mutations in our previous study (C228T and C250T) is documented in Table III (12). The individuals carrying rs2853669 variant allele $(\mathrm{CT}+\mathrm{CC})$ was found in $66.7 \%(20 / 30)$ of TERT promoter mutation-positive patients and this interaction did not increase the risk $(\mathrm{OR}=1.54$; 95\% $\mathrm{CI}=0.62-3.67 ; \mathrm{P}=0.33$ ). Furthermore, the distribution of the TERT rs2853669 polymorphism among patients with high-risk HPV also did not increase the risk of cervical cancer (OR=1.54; 95\% CI=0.79-3.01; $\mathrm{P}=0.203$; Table IV).

Meta-analysis results. Based on the inclusion criteria, a total of 17 studies from 14 previously published articles (15,23-35) along with the present study results were analyzed for the association between the TERT rs2583669 polymorphism and cancer susceptibility; this included 9,537 cancer cases and 12,370 controls (Table V). By pooling all the previous studies, a statistically significant association between the TERT rs2583669 polymorphism and cancer risk was identified (dominant model: Pooled OR=1.09; 95\% CI, 1.03-1.16; $\mathrm{P}=0.004$; Fig. 2). Further, stratification analyses were performed to assess the risk by type of cancer and ethnicity (Table SI). TERT rs2583669 polymorphism and cancer risk was not statistically significant in different ethnicities. Stratification analyses by type of cancer showed significant association of TERT rs2583669 polymorphism with acute myeloid leukemia, hepatocellular carcinoma and lung cancer (Table SI). Significant heterogeneity was observed in all genetic models tested (Table SI). Sensitivity analysis performed by omitting individual studies revealed that there was no change in the pooled ORs (Fig. 3). The shape of the funnel plot (Fig. 4) and Egger's tests did not reveal any evidence for asymmetry in all three genetic models (Table SI).

\section{Discussion}

TERT has been found to be overexpressed in $90 \%$ of human cancer (36). Furthermore, genetic alterations in the proximal promoter of TERT were shown to be significantly associated with a range of clinical stages of different cancer types (37). Recently, one of the mechanisms of TERT regulation through the non-coding driver mutations (C228T and C250T) in the TERT promoter has been reported in several cancer types with different frequencies (38-40). The mutations created a new binding site for the ETS family of transcription factors, resulting in the overexpression of TERT $(9,10)$. However, the mechanism by which it overexpresses and the length of the telomere were reported to be different in different types of tumor. TERT has been shown to co-operate with activated oncogenes and inactivated tumor suppressor genes in 
Table II. Association between TERT rs2853669 polymorphism and cervical cancer.

\begin{tabular}{|c|c|c|c|c|c|}
\hline TERT SNP rs2853669 A>G & $\begin{array}{l}\text { Cervical cancer }(n=257) \\
\text { (Percentage frequency) }\end{array}$ & $\begin{array}{c}\text { Control }(\mathrm{n}=295) \\
\text { (Percentage frequency) }\end{array}$ & OR & $95 \% \mathrm{CI}$ & P-value \\
\hline \multicolumn{6}{|l|}{ Genotype } \\
\hline AA & $60(23.3)$ & $63(21.4)$ & & Reference & \\
\hline $\mathrm{AG}$ & $91(35.4)$ & $146(49.5)$ & 0.66 & $0.42-1.02$ & 0.058 \\
\hline GG & $106(41.3)$ & $86(29.1)$ & 1.29 & $0.82-2.04$ & 0.265 \\
\hline Dominant model AG+GG vs. AA & $197(76.7)$ & $232(78.6)$ & 0.89 & $0.60-1.33$ & 0.575 \\
\hline Recessive model GG vs. AA+AG & $106(41.3)$ & $86(29.1)$ & 1.71 & $1.20-2.43$ & 0.003 \\
\hline \multicolumn{6}{|l|}{ Allelic model } \\
\hline A & $211(41.1)$ & $272(46.1)$ & & Reference & \\
\hline G & $303(58.9)$ & $318(53.9)$ & 1.23 & $0.97-1.56$ & 0.091 \\
\hline HWE $\chi^{2}$ & 18.51 & 0.005 & & & \\
\hline HWE P-value & $<0.001$ & 0.944 & & & \\
\hline
\end{tabular}

ORs with the 95\% CIs were calculated using SPSS. TERT, telomerase reverse transcriptase; SNP, single nucleotide polymorphism; OR, odds ratio; HWE, Hardy Weinberg Equilibrium; CI, confidence interval.

Table III. Association of TERT promoter mutations (C228T and C250T) with rs2853669 (-245T/C) in cervical cancer from our previous study (12).

TERT mutation status

\begin{tabular}{lcccc}
\cline { 2 - 3 } SNP rs2853669T>C (n=140) & Wild-type & Mutated & OR (95\% CI) & P-value \\
\hline TT (37) & 27 & 10 & & Reference \\
CT+CC (103) & 83 & 20 & $1.54(0.62-3.67)$ & 0.333 \\
\hline
\end{tabular}

ORs with 95\% CIs were calculated using SPSS. TERT, telomerase reverse transcriptase; SNP, single nucleotide polymorphism; OR, odds ratio; CI, confidence interval.

Table IV. Association of HPV and telomerase reverse transcriptase rs2853669 (-245T/C) in cervical cancer.

\begin{tabular}{|c|c|c|c|c|}
\hline \multirow[b]{2}{*}{ SNP rs2853669T $>C \quad(n=257)$} & \multicolumn{2}{|c|}{ HPV status } & \multirow[b]{2}{*}{ OR $(95 \% \mathrm{CI})$} & \multirow[b]{2}{*}{ P-value } \\
\hline & Positive (\%) & Negative $(\%)$ & & \\
\hline TT (60) & $46(76.7)$ & $14(23.3)$ & & \\
\hline CT+CC (197) & $134(68.0)$ & $63(32.0)$ & $1.54(0.79-3.01)$ & 0.203 \\
\hline
\end{tabular}

ORs with 95\% CIs were calculated using SPSS. HPV, human papilloma virus; SNP, single nucleotide polymorphism; OR, odds ratio; CI, confidence interval.

tumorigenesis (41). In addition, viral oncogene expression and other genetic alterations, including mutations and SNPs in both coding and non-coding regions, were shown to play a major role in carcinogenesis (42). Advances in next generation sequencing resulted in the identification of several SNPs and mutations in non-coding regulatory regions, highlighting the role of genetic variants in the regulatory region of genes in tumorigenesis (8).

The TERT promoter SNP rs2853669 and its association with cancer risk has been reported in various cancer types in different populations (13-15). However, the role of this particular SNP with the TERT promoter driver mutations and its genetic association with HPV in patients with cervical cancer had not yet been studied, to the best of our knowledge. In the present study, to understand the genetic association of the TERT SNP with cervical cancer risk, the SNP rs2853669 was genotyped in 257 cervical cancer samples of South Indian origin and 295 control samples, and a higher frequency of the rs2853669 variant allele $(\mathrm{G})$ was observed in the cervical cancer samples compared with the control samples. The SNP rs2853669 in the TERT promoter has been shown to have a functional impact on TERT regulation and telomere length 
Table V. Telomerase reverse transcriptase rs2853669 and cancer association studies included in the meta-analysis.

\begin{tabular}{|c|c|c|c|c|c|c|c|c|c|c|c|c|}
\hline \multirow[b]{2}{*}{ Author, year } & \multirow[b]{2}{*}{ Serial no. } & \multirow[b]{2}{*}{ Disease } & \multirow[b]{2}{*}{ Country } & \multirow[b]{2}{*}{ Ethnicity } & \multicolumn{3}{|c|}{ Cases } & \multicolumn{3}{|c|}{ Control } & \multirow{2}{*}{$\frac{\text { HWE }}{\text { P-value }}$} & \multirow[b]{2}{*}{ (Refs.) } \\
\hline & & & & & $\mathrm{CC}$ & $\mathrm{CT}$ & $\mathrm{TT}$ & $\mathrm{CC}$ & $\mathrm{CT}$ & $\mathrm{TT}$ & & \\
\hline Present Study & 1 & Cervical cancer & India & Asian & 106 & 91 & 60 & 86 & 146 & 63 & 0.944 & - \\
\hline Zhang et al, 2017 & 2 & Gastric cancer & China & Asian & 44 & 136 & 180 & 36 & 219 & 473 & 0.109 & (23) \\
\hline Xing et al, 2016 & 3 & Lung cancer & China & Asian & 41 & 162 & 215 & 30 & 145 & 235 & 0.249 & $(24)$ \\
\hline Bayram et al, 2016 & 4 & Gastric cancer & Turkey & Caucasian & 13 & 47 & 44 & 35 & 99 & 75 & 0.810 & (25) \\
\hline Oztas et al, 2016 & 5 & Breast cancer & Turkey & Caucasian & 24 & 47 & 36 & 25 & 52 & 31 & 0.723 & (26) \\
\hline Jannuzzi et al, 2015 & 6 & Colorectal cancer & Turkey & Caucasian & 15 & 50 & 31 & 17 & 58 & 40 & 0.587 & $(27)$ \\
\hline Mosrati et al, 2015 & 7 & Glioblastoma & Sweden & Caucasian & 11 & 48 & 69 & 65 & 341 & 373 & 0.293 & (15) \\
\hline Mosrati et al, 2015 & 8 & AML & Sweden & Caucasian & 38 & 99 & 89 & 65 & 341 & 373 & 0.293 & $(15)$ \\
\hline Yoo et al, 2015 & 9 & Lung cancer & Korea & Asian & 137 & 477 & 478 & 105 & 490 & 485 & 0.242 & (28) \\
\hline Shadrina et al, 2015 & 10 & NHL & Russia & Caucasian & 35 & 213 & 272 & 71 & 322 & 491 & 0.079 & $(29)$ \\
\hline Zhong et al, 2013 & 11 & Lung cancer & China & Asian & 108 & 242 & 148 & 72 & 224 & 206 & 0.381 & $(30)$ \\
\hline Liu et al, 2011 & 12 & SCCHN & USA & Caucasian & 79 & 381 & 428 & 85 & 375 & 425 & 0.863 & $(31)$ \\
\hline Shen et al, 2010 & 13 & Breast cancer & USA & Caucasian & 86 & 445 & 503 & 128 & 432 & 522 & 0.009 & $(32)$ \\
\hline Park et al, 2010 & 14 & $\mathrm{HCC}$ & Korea & Asian & 35 & 121 & 134 & 68 & 110 & 99 & 0.001 & $(33)$ \\
\hline Varadi et al, 2009 & 15 & Breast cancer & Poland & Caucasian & 58 & 299 & 411 & 38 & 154 & 244 & 0.059 & (34) \\
\hline Varadi et al, 2009 & 16 & Breast cancer & Sweden & Caucasian & 47 & 310 & 409 & 143 & 558 & 818 & 0.001 & (34) \\
\hline Savage et al, 2007 & 17 & Breast cancer & Poland & Caucasian & 1,095 & 766 & 124 & 1,224 & 900 & 158 & 0.669 & $(35)$ \\
\hline
\end{tabular}

AML, acute myeloid leukemia; NHL, non-Hodgkin lymphoma; SCCHN, squamous cell carcinoma of the head and neck; HCC, hepatocellular carcinoma; HWE, Hardy-Weinberg equilibrium.

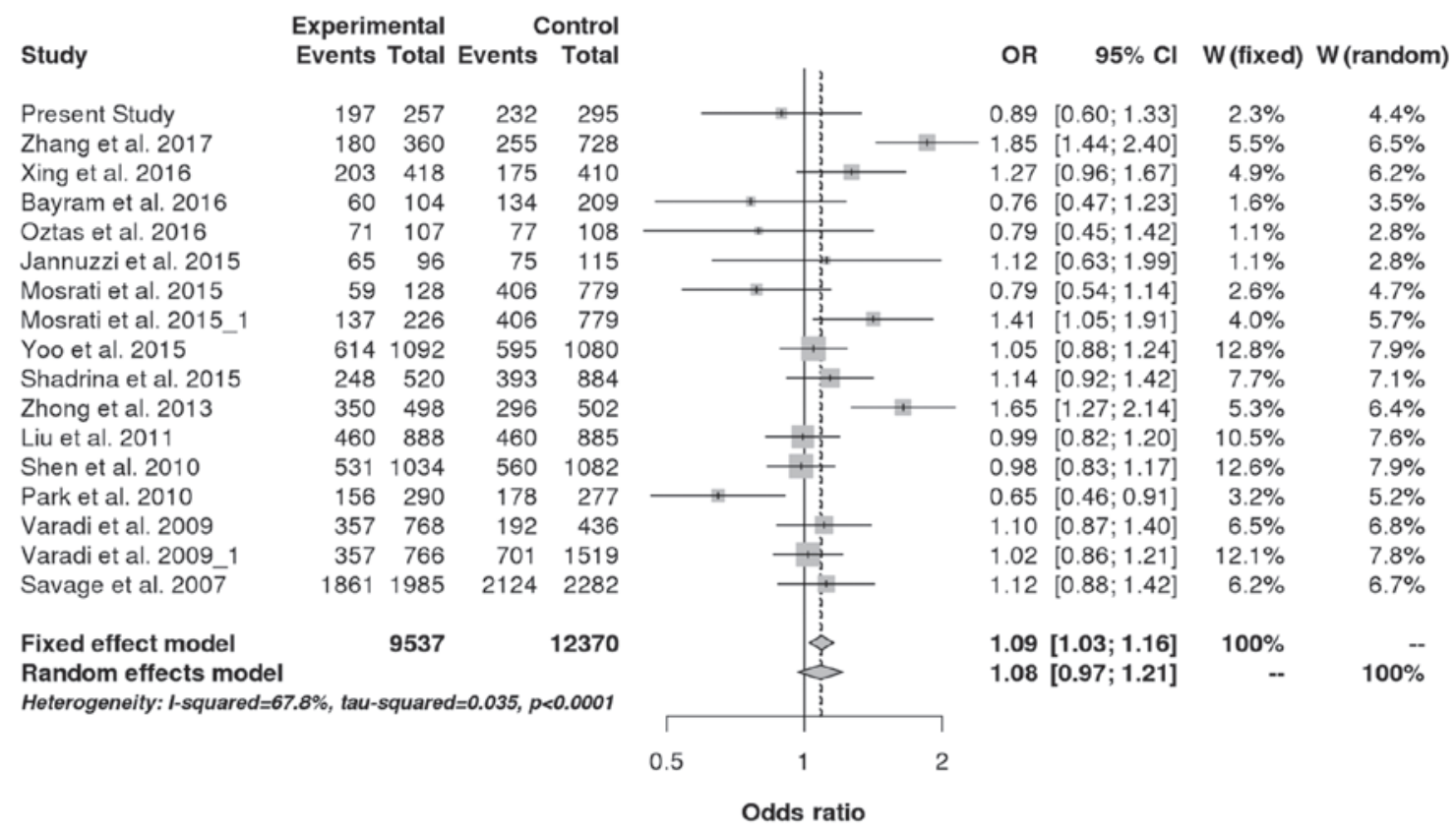

Figure 2. Meta-analysis for the association between various cancer types and telomerase reverse transcriptase rs2853669 polymorphism. Reference numbers of the respective studies are provided in Table V. The squares indicate the ORs in each study, with square sizes inversely proportional to the standard error of the OR. The diamond shape indicates the pooled ORs. Horizontal lines represent $95 \%$ CI. OR, odds ratio.

in various cancer types $(34,35,43,44)$. Furthermore, the genetic association of the TERT SNP rs2853669 has been reported in many cancer types of different populations where the disease association was different between the population and the cancer types. The variant rs2853669 is located in the binding site of ETS-2, another transcription factor of the ETS family, which regulates various genes involved in cellular senescence and tumorigenesis $(45,46)$. The results of either the SNP of TERT (rs2853669) alone or the modifying effect of rs2853669 on TERT hotspot promoter mutations have been shown to 
Study

Omitting Present Study Omitting Zhang et al. 2017

Omitting Xing et al. 2016 Omitting Bayram et al. 2016 Omitting Oztas et al. 2016

Omitting Jannuzzi et al. 2015 Omitting Mosrati et al. 2015 Omitting Mosrati et al. 2015 Omitting Yoo et al. 2015 Omitting Shadrina et al. 2015 Omitting Zhong et al. 2013 Omitting Liu et al. 2011 Omitting Shen et al. 2010 Omitting Park et al. 2010 Omitting Varadi et al. 2009 Omitting Varadi et al. 2009 Omitting Savage et al. 2007

Fixed effect mode

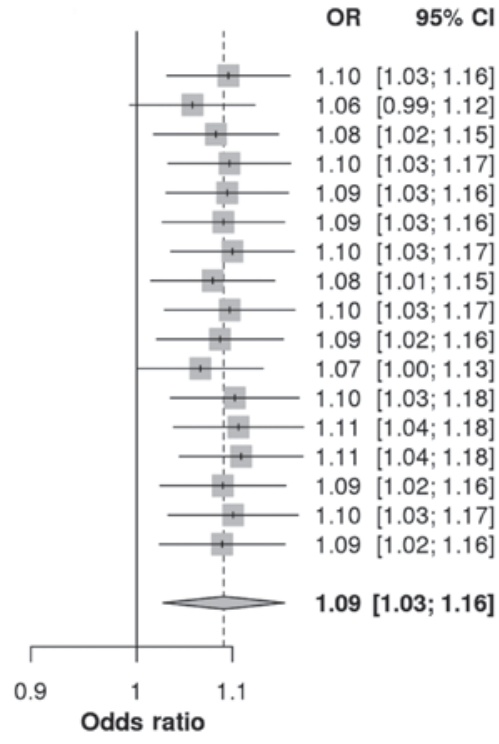

Figure 3. Sensitivity analysis of meta-analysis results of telomerase reverse transcriptase rs2853669 polymorphism. Sensitivity analysis was performed to generate a forest plot by excluding one of the studies in each time. Reference numbers of the respective studies are presented in Table V. The squares indicate the ORs after omitting each study and the diamond shape indicates the pooled ORs. Horizontal lines represent $95 \%$ CI. OR, odds ratio.

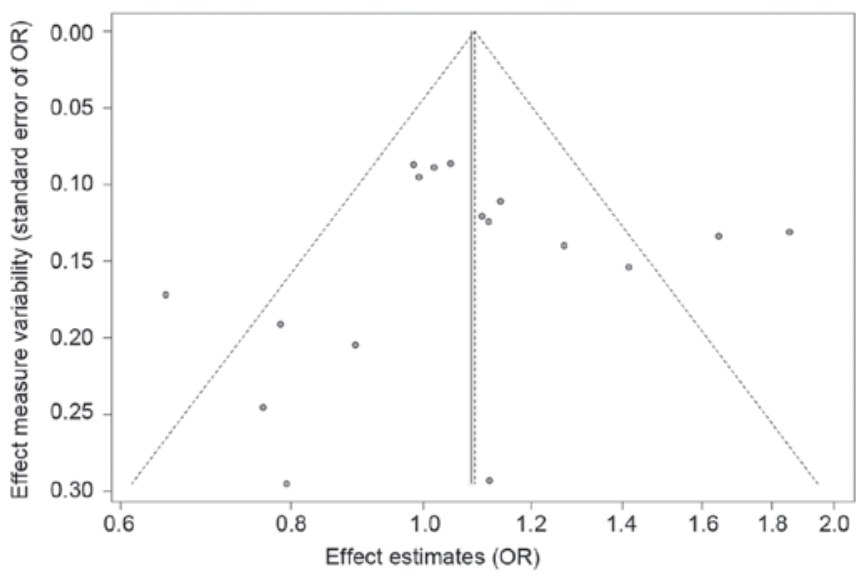

Figure 4. Funnel plot used in assessing publication bias in the meta-analysis of telomerase reverse transcriptase rs 2853669 . The dotted vertical line indicates the triangular region within which $95 \%$ of studies are expected to lie in the absence of bias and heterogeneity. The solid vertical line corresponds to no intervention effect. OR, odds ratio.

be highly controversial with varying results across different previous studies $(15,31,34,35,44,47,48)$.

The combination of the rs2853669 variant allele $(\mathrm{G})$ and TERT promoter hotspot mutations was also high compared with hotspot mutations and the wild-type allele. Almost all the patients with the rs2853669 and hotspot mutations were observed in poorly differentiated squamous cell carcinoma tumors except one patient where it was a moderately differentiated tumor (49). It has been reported that the TERT hotspot mutation-positive cases showed poor survival in the absence of the variant allele (47). On the contrary, previous studies on glioblastoma showed a shortest mean overall survival, which was mainly detected in patients harboring both an activating TERT promoter mutation and the rs2853669 variant homozygous allele $(14,15)$. Moreover, the role of rs2853669 with or without TERT hotspot mutations on TERT regulation and activation has been demonstrated through functional experiments in hepatocellular carcinoma (HCC) cell lines. E2F1 binding to the TERT promoter can enhance interaction with other epigenetic modifiers like DNMT1 and HDAC1 to enable promoter methylation-mediated regulation of TERT transcription, which suggested that E2F1 could potentially function as a transcriptional repressor of TERT (16). The SNP (rs2853669) may interfere with the binding of E2F1 and influence promoter methylation associated with the TERT transcription as the SNP resides at $2 \mathrm{bp}$ downstream of E2F1 binding site on the TERT promoter (16). Recently, the combination of $-124 \mathrm{C}>\mathrm{T}(\mathrm{C} 228 \mathrm{~T})$ mutation and $\mathrm{rs} 2853669(-245 \mathrm{~T}>\mathrm{C})$ variant was reported to be correlated with increased TERT transcription activity in $\mathrm{HCC}$ cases that frequently resulted in higher risk of recurrence (16). The increased mortality in HCC has been observed in cases with the co-existence of the two hotspot TERT promoter mutations and SNPs (16). The expression level of TERT is increased by the inhibition of the transcriptional repressor E2F1 in the presence of the variant allele $-245 \mathrm{~T}>\mathrm{C}$, together with the activation of the ETS2 transcription factor due to the $-124 \mathrm{C}>\mathrm{T}$ mutation. A recent study reported that the $-245 \mathrm{~T}$ region located on an ETS2 binding site is not a native ETS2 binding site, suggesting that the TERT promoter mutation $(-124 \mathrm{C}>\mathrm{T}$ and $-146 \mathrm{C}>\mathrm{T})$ cooperates with its native ETS binding sites to form high-order structures such as G-quadruplexes, contributing to the recruitment rs2853669 with the non-coding driver hotspot mutation in the TERT promoter has been shown to be associated with poor prognosis in HCC (16). Furthermore, the present results suggested that the hotspot TERT promoter mutations and the SNP combinations might play an important role in TERT regulation. In addition, the expression of TERT was shown to be regulated by HPV E6 oncoprotein $(50,51)$. The combination of the rs 2853669 variant allele and TERT promoter hotspot mutation (C228T and C250T) along with HPV could be an additional risk to patients with cervical cancer. However, the present study did not identify any significant association between the TERT SNP (rs2853669) and the presence of HPV.

In addition, to understand and compare the genotype frequency and the risk associated with the SNP rs2853669 among various cancer types and different populations, a meta-analysis was conducted. Due to the lack of previous studies on the association of rs2853669 and cervical cancer risk in different populations, a meta-analysis using different cancer types of the world population was conducted. In the present study, the variant allele ' $G$ ' showed a significantly increased association with cancer risk in the dominant model. This result was consistent with previous studies reported from other human cancer types and this result was also obtained in the present meta-analysis $(14,28,29,46)$. Overall, the present meta-analysis showed a significant risk association of the SNP rs2853669 with various cancer types of the different ethnic population. In contrast, Shen et al (52) reported that the SNP had no association with cancer risk and prognosis. The number and ethnic origin of the samples could influence the outcome of the results. However, when the SNP was combined with TERT promoter mutations, a modifying 
effect of rs2853669 among patients with cancer with TERT promoter mutations was observed and only those patients carrying the TT genotype had a poor survival (52). Although the effect of both the SNP and the TERT promoter mutations were analyzed in the present study, it was not possible to identify an association between the TERT SNP with the clinicopathological features of the patients with cervical cancer, as the majority of the patient history showed poor follow up and/or drop out from the treatments. Moreover, all previous genome-wide association studies (GWAS) showed an association of this SNP with telomere length (53). To the best of our knowledge, no previous GWAS study has reported the association of SNP with cancer risk/clinical features, although many individual previous studies have reported the association of this SNP with cancer risk across human cancer but not in cervical cancer (14-16,33).

In conclusion, the present study suggests that the TERT rs2853669 variant ' $G G$ ' may play a role in the progression of cervical cancer in South India as well in different cancer types of world populations. The present study enrolled female patients from a relatively homogenous population of South India; however, the limitations of the present study should be considered when interpreting the results. A major limitation of the present study is the collection of tissue from cases and blood samples from controls for DNA extraction and genotyping. Furthermore, a number of demographic and clinicopathological characteristics limited the present study. Owing to inadequate data, the impact of demographic and clinicopathological characteristics was not considered to perform multivariate analysis. Furthermore, non-availability of the data on Helicobacter pylori infection, alcohol consumption and smoking, limited the evaluation of the potential interactions between these risk factors and TERT rs2853669 polymorphism. In conclusion, within the limitations, the present study provides an insight on the significance of genetic variants present in the non-coding regions of genes and their association with the hotspot mutations. However, functional studies are warranted to establish the role of rs2853669 in cervical carcinogenesis.

\section{Acknowledgements}

Not applicable.

\section{Funding}

The present study was supported by The University Grants Commission (UGC-SAP) and Department of Science and Technology (DST-FIST) grants and the Department of Health Research, DHR-MRU grant, Government of India for the research infrastructural facilities. Research fellowships were awarded to VV and KA from Council of Scientific and Industrial Research, and SR and GA from University Grants Commission, New Delhi, Government of India. The present study was supported by research grants from Department of Atomic Energy, Board of Research in Nuclear Sciences, Mumbai, India (grant no. 35/14/10/2014-BRNS/0210) and Department of Biotechnology, New Delhi, India (grant no. BT/PR10023/AGR/36/27/2007) to AKM.

\section{Availability of data and materials}

The datasets used and/or analyzed during the present study are available from the corresponding author on reasonable request.

\section{Authors' contributions}

AKMun conceived the study, designed the experiments and supervised the study. VV and SR contributed to the study design, performed the experiments, analyzed the data and drafted the manuscript. KA and GA helped conduct the experiments and drafted the manuscript. RR provided tumor samples and clinical data. AKMur, LVKSB and AKMun analyzed the data and critically reviewed the manuscript. All authors read and approved the final manuscript.

\section{Ethics approval and consent to participate}

The present study was conducted after obtaining approval from The Institutional Ethics Committee (approval no. 04092010) of Madras Medical College and Hospital (Chennai, China). The tissue/blood samples were collected from The Institute of Social Obstetrics and Government Kasturba Gandhi Hospital for Women and Children, Triplicane, Chennai and Government Royapettah Hospital after obtaining written informed consent from each patient.

\section{Patient consent for publication}

Not applicable.

\section{Competing interests}

The authors declare that they have no competing interests.

\section{References}

1. Low KC and Tergaonkar V: Telomerase: Central regulator of all of the hallmarks of cancer. Trends Biochem Sci 38: 426-434, 2013.

2. Maciejowski $\mathbf{J}$ and de Lange T: Telomeres in cancer: Tumour suppression and genome instability. Nat Rev Mol Cell Biol 18: 175-186, 2017.

3. Pestana A, Vinagre J, Sobrinho-Simões M and Soares P: TERT biology and function in cancer: Besyond immortalisation. $\mathrm{J}$ Mol Endocrinol 58: R129-R146, 2017.

4. Barthel FP, Wei W, Tang M, Martinez-Ledesma E, Hu X, Amin SB, Akdemir KC, Seth S, Song X, Wang Q, et al: Systematic analysis of telomere length and somatic alterations in 31 cancer types. Nat Genet 49: 349-357, 2017.

5. Sreedevi A, Javed R and Dinesh A: Epidemiology of cervical cancer with special focus on India. Int J Womens Health 7: 405-414, 2015.

6. Schiffman M, Wentzensen N, Wacholder S, Kinney W, Gage JC and Castle PE: Human papillomavirus testing in the prevention of cervical cancer. J Natl Cancer Inst 103: 368-383, 2011.

7. Cancer Genome Atlas Research Network; Albert Einstein College of Medicine; Analytical Biological Services; Barretos Cancer Hospital; Baylor College of Medicine; Beckman Research Institute of City of Hope; Buck Institute for Research on Aging; Canada's Michael Smith Genome Sciences Centre; Harvard Medical School; Helen F. Graham Cancer Center \& Research Institute at Christiana Care Health Services, et al: Integrated genomic and molecular characterization of cervical cancer. Nature 543: 378-384, 2017.

8. Khurana E, Fu Y, Chakravarty D, Demichelis F, Rubin MA and Gerstein M: Role of non-coding sequence variants in cancer. Nat Rev Genet 17: 93-108, 2016. 
9. Horn S, Figl A, Rachakonda PS, Fischer C, Sucker A, Gast A, Kadel S, Moll I, Nagore E, Hemminki K, et al: TERT promoter mutations in familial and sporadic melanoma. Science 339: 959-961, 2013.

10. Liu X, Bishop J, Shan Y, Pai S, Liu D, Murugan AK, Sun H, El-Naggar AK and Xing M: Highly prevalent TERT promoter mutations in aggressive thyroid cancers. Endocr Relat Cancer 20: 603-610, 2013

11. Killela PJ, Reitman ZJ, Jiao Y, Bettegowda C, Agrawal N, Diaz LA Jr, Friedman AH, Friedman H, Gallia GL, Giovanella BC, et al: TERT promoter mutations occur frequently in gliomas and a subset of tumors derived from cells with low rates of self-renewal. Proc Natl Acad Sci USA 110: 6021-6026, 2013.

12. Vinothkumar V, Arunkumar G, Revathidevi S, Arun K, Manikandan M, Rao AK, Rajkumar KS, Ajay C, Rajaraman R, Ramani R, et al: TERT promoter hot spot mutations are frequent in Indian cervical and oral squamous cell carcinomas. Tumour Biol 37: 7907-7913, 2016

13. Park CK, Lee SH, Kim JY, Kim JE, Kim TM, Lee ST, Choi SH, Park SH and Kim IH: Expression level of hTERT is regulated by somatic mutation and common single nucleotide polymorphism at promoter region in glioblastoma. Oncotarget 5: 3399-3407, 2014.

14. Spiegl-Kreinecker S, Lotsch D, Ghanim B, Pirker C, Mohr T, Laaber M, Weis S, Olschowski A, Webersinke G, Pichler J and Berger W: Prognostic quality of activating TERT promoter mutations in glioblastoma: Interaction with the rs2853669 polymorphism and patient age at diagnosis. Neuro Oncol 17: $1231-1240,2015$

15. Mosrati MA, Malmström A, Lysiak M, Krysztofiak A, Hallbeck M, Milos P, Hallbeck AL, Bratthäll C, Strandéus M, Stenmark-Askmalm $M$ and Söderkvist P: TERT promoter mutations and polymorphisms as prognostic factors in primary glioblastoma. Oncotarget 6: 16663-16673, 2015.

16. Ko E, Seo HW, Jung ES, Kim BH and Jung G: The TERT promoter SNP rs2853669 decreases E2F1 transcription factor binding and increases mortality and recurrence risks in liver cancer. Oncotarget 7: 684-699, 2016.

17. Ostör AG: Natural history of cervical intraepithelial neoplasia: A critical review. Int J Gynecol Pathol 12: 186-192, 1993.

18. Green MR: Molecular Cloning: A Laboratory Manual. Green MR and Sambrook J (eds). Cold Spring Harbor Laboratory Press, New York, NY, 2012.

19. Chen B, Cole JW and Grond-Ginsbach C: Departure from Hardy Weinberg equilibrium and genotyping error. Front Genet 8: 167, 2017.

20. Moher D, Liberati A, Tetzlaff J and Altman DG: Preferred reporting items for systematic reviews and meta-analyses: The PRISMA statement. PLoS Med 6: e1000097, 2009.

21. Wallace BC, Dahabreh IJ, Trikalinos TA, Lau J, Trow P, Schmid CH. Closing the gap between methodologists and end-users: R as a computational back-end. J Stat Software 49: 15,2012 .

22. Egger M, Davey Smith G, Schneider M and Minder C: Bias in meta-analysis detected by a simple, graphical test. BMJ 315 : 629-634, 1997.

23. Zhang J, Ju H, Gao JR, Jiao XL and Lu Y: Polymorphisms in human telomerase reverse transcriptase (hTERT) gene, gene-gene and gene-smoking interaction with susceptibility to gastric cancer in Chinese Han population. Oncotarget 8 : 20235-20243, 2017.

24. Xing YL, Liu F, Li JF, Lin JC, Zhu GD, Li M, Zhang CR and Niu YY: Case-control study on impact of the telomerase reverse transcriptase gene polymorphism and additional single nucleotide polymorphism (SNP)-SNP interaction on non-small cell lung cancers risk in Chinese Han population. J Clin Lab Anal 30: 1071-1077, 2016

25. Bayram S, Ülger Y, Sümbül AT, Kaya BY, Genc A, Rencüzoğullari E and Dadaş E: Polymorphisms in human telomerase reverse transcriptase (hTERT) gene and susceptibility to gastric cancer in a Turkish population: Hospital-based case-control study. Gene 585: 84-92, 2016.

26. Oztas E, Kara H, Kara ZP, Aydogan MU, Uras C and Ozhan G: Association between human telomerase reverse transcriptase gene variations and risk of developing breast cancer. Genet Test Mol Biomarkers 20: 459-464, 2016.

27. Jannuzzi AT, Karaman E, Oztas E, Yanar HT and Özhan G: Telomerase reverse transcriptase (TERT) gene variations and susceptibility of colorectal cancer. Genet Test Mol Biomarkers 19: 692-697, 2015.
28. Yoo SS, Do SK, Choi JE, Lee SY, Lee J, Cha SI, Kim CH and Park JY: TERT polymorphism rs2853669 influences on lung cancer risk in the Korean population. J Korean Med Sci 30: $1423-1428,2015$.

29. Shadrina AS, Boyarskikh UA, Oskina NA, Sinkina TV, Lazarev AF, Petrova VD and Filipenko ML: TERT polymorphisms rs2853669 and rs7726159 influence on prostate cancer risk in Russian population. Tumour Biol 36: 841-847, 2015.

30. Zhong R, Liu L, Zou L, Zhu Y, Chen W, Zhu B, Shen N, Rui R, Long L, Ke J, et al: Genetic variations in TERT-CLPTM1L locus are associated with risk of lung cancer in Chinese population. Mol Carcinog 52 (Suppl 1): E118-E126, 2013.

31. Liu Z, Ma H, Wei S, Li G, Sturgis EM and Wei Q: Telomere length and TERT functional polymorphisms are not associated with risk of squamous cell carcinoma of the head and neck. Cancer Epidemiol Biomarkers Prev 20: 2642-2645, 2011.

32. Shen J, Gammon MD, Wu HC, Terry MB, Wang Q, Bradshaw PT, Teitelbaum SL, Neugut AI and Santella RM: Multiple genetic variants in telomere pathway genes and breast cancer risk. Cancer Epidemiol Biomarkers Prev 19: 219-228, 2010.

33. Park J, Chang H, Ahn S, Lee J, Kim D, Lee K, Chon C, Moon Y and Han K: hTERT promoter gene polymorphism and the risk of hepatocellular carcinoma (HCC) in patients with chronic hepatitis B. J Hepatol 52 (Suppl 1): S345, 2010.

34. Varadi V, Brendle A, Grzybowska E, Johansson R, Enquist K, Butkiewicz D, Pamula-Pilat J, Pekala W, Hemminki K, Lenner P and Försti A: A functional promoter polymorphism in the TERT gene does not affect inherited susceptibility to breast cancer. Cancer Genet Cytogenet 190: 71-74, 2009.

35. Savage SA, Chanock SJ, Lissowska J, Brinton LA, Richesson D, Peplonska B, Bardin-Mikolajczak A, Zatonski W, Szeszenia-Dabrowska N and Garcia-Closas M: Genetic variation in five genes important in telomere biology and risk for breast cancer. Br J Cancer 97: 832-836, 2007.

36. Jiang Y, Chen C, Chen SM, Wang YQ, Xu Y, Wang Y, Chen Z, Xiao BK and Tao ZZ: Telomerase reverse transcriptase promotes the proliferation of human laryngeal carcinoma cells through activation of the activator protein 1. Oncol Lett 6: 75-80, 2013.

37. Shay JW: Role of telomeres and telomerase in aging and cancer. Cancer Discov 6: 584-593, 2016.

38. Liu R and Xing M: TERT promoter mutations in thyroid cancer. Endocr Relat Cancer 23: R143-R155, 2016.

39. Shimoi T, Yoshida M, Kitamura Y, Yoshino T, Kawachi A, Shimomura A, Noguchi E, Yunokawa M, Yonemori K, Shimizu C, et al: TERT promoter hotspot mutations in breast cancer. Breast Cancer 25: 292-296, 2018.

40. Thiem S, Herold T, Krafft U, Bremmer F, Tolkach Y, Szász AM, Kriegsmann J, Gaisa NT, Niedworok C, Szarvas T and Reis H: Telomerase reverse transcriptase (TERT) promoter mutations are rare in urachal cancer. Pathol Int 67: 597-601, 2017.

41. Liu X, Dakic A, Chen R, Disbrow GL, Zhang Y, Dai Y and Schlegel R: Cell-restricted immortalization by human papillomavirus correlates with telomerase activation and engagement of the hTERT promoter by Myc. J Virol 82: 11568-11576, 2008.

42. Chen Y, Williams V, Filippova M, Filippov V and Duerksen-Hughes P: Viral carcinogenesis: Factors inducing DNA damage and virus integration. Cancers (Basel) 6: 2155-2186, 2014

43. Hsu CP, Hsu NY, Lee LW and Ko JL: Ets2 binding site single nucleotide polymorphism at the hTERT gene promoter-effect on telomerase expression and telomere length maintenance in non-small cell lung cancer. Eur J Cancer 42: 1466-1474, 2006.

44. Bojesen SE, Pooley KA, Johnatty SE, Beesley J, Michailidou K, Tyrer JP, Edwards SL, Pickett HA, Shen HC, Smart CE, et al: Multiple independent variants at the TERT locus are associated with telomere length and risks of breast and ovarian cancer. Nat Genet 45: 371-384, 384e1-2, 2013.

45. Horikawa I, Cable PL, Afshari C and Barrett JC: Cloning and characterization of the promoter region of human telomerase reverse transcriptase gene. Cancer Res 59: 826-830, 1999.

46. Helbig S, Wockner L, Bouendeu A, Hille-Betz U, McCue K, French JD, Edwards SL, Pickett HA, Reddel RR, Chenevix-Trench G, et al: Functional dissection of breast cancer risk-associated TERT promoter variants. Oncotarget 8: 67203-67217, 2017.

47. Rachakonda PS, Hosen I, de Verdier PJ, Fallah M, Heidenreich B, Ryk C, Wiklund NP, Steineck G, Schadendorf D, Hemminki K and Kumar R: TERT promoter mutations in bladder cancer affect patient survival and disease recurrence through modification by a common polymorphism. Proc Natl Acad Sci USA 110: 17426-17431, 2013 
48. Nagore E, Heidenreich B, Requena C, García-Casado Z, Martorell-Calatayud A, Pont-Sanjuan V, Jimenez-Sanchez AI and Kumar R: TERT promoter mutations associate with fast-growing melanoma. Pigment Cell Melanoma Res 29: 236-238, 2016

49. Bell RJA, Rube HT, Xavier-Magalhães A, Costa BM, Mancini A, Song JS and Costello JF: Understanding TERT promoter mutations: A common path to immortality. Mol Cancer Res 14: 315-323, 2016.

50. McMurray HR and McCance DJ: Human papillomavirus type 16 E6 activates TERT gene transcription through induction of c-Myc and release of USF-mediated repression. J Virol 77: 9852-9861, 2003.
51. Liu X, Dakic A, Zhang Y, Dai Y, Chen R and Schlegel R: HPV E6 protein interacts physically and functionally with the cellular telomerase complex. Proc Natl Acad Sci USA 106: 18780-18785, 2009.

52. Shen N, Lu Y, Wang X, Peng J, Zhu Y and Cheng L: Association between rs2853669 in TERT gene and the risk and prognosis of human cancer: A systematic review and meta-analysis. Oncotarget 8: 50864-50872, 2017.

53. Soerensen $M$, Thinggaard M, Nygaard M, Dato S, Tan Q, Hjelmborg J, Andersen-Ranberg K, Stevnsner T, Bohr VA, Kimura M, et al: Genetic variation in TERT and TERC and human leukocyte telomere length and longevity: A cross-sectional and longitudinal analysis. Aging Cell 11: 223-227, 2012. 